\title{
Distributions of Conductance and Shot Noise and Associated Phase Transitions
}

\author{
Pierpaolo Vivo ${ }^{1}$, Satya N. Majumdar ${ }^{2}$ and Oriol Bohigas ${ }^{2}$ \\ 1 Department of Mathematical Sciences,Brunel University, \\ Uxbridge, Middlesex, UB8 3PH (United Kingdom) \\ 2 Laboratoire de Physique Théorique et Modèles Statistiques (UMR 8626 du CNRS), \\ Université Paris-Sud, Bâtiment 100, 91405 Orsay Cedex (France)
}

(Dated: October 31, 2018)

\begin{abstract}
For a chaotic cavity with two indentical leads each supporting $N$ channels, we compute analytically, for large $N$, the full distribution of the conductance and the shot noise power and show that in both cases there is a central Gaussian region flanked on both sides by non-Gaussian tails. The distribution is weakly singular at the junction of Gaussian and non-Gaussian regimes, a direct consequence of two phase transitions in an associated Coulomb gas problem.
\end{abstract}

PACS numbers: 73.23.-b, 02.10.Yn, 24.60.-k, 21.10.Ft

Ballistic transport of electrons through a mesoscopic cavity such as a quantum dot has been studied intensively both theoretically and experimentally in recent times [1]. In the simplest setting one considers a single cavity of submicron dimension (e.g., a quantum dot) with two identical leads (each supporting $N$ channels) connecting it to two separate electron reservoirs. An electron, injected through one lead, gets scattered in the cavity and leaves by either of the two leads. The transport of electrons through such an open quantum system is encoded in the $2 N \times 2 N$ unitary scattering matrix $S=\left(\begin{array}{cc}r & t^{\prime} \\ t & r^{\prime}\end{array}\right)$ connecting the incoming and outgoing electron wavefunctions, where $r, t$ are $N \times N$ reflection and transmission matrices from the left and $r^{\prime}, t^{\prime}$ from the right. Several experimentally measurable transport observables such as the conductance (i.e., the time averaged current) and the shot noise power (that describes the current fluctuations associated with the granularity of electronic charge $e$ ) can be expressed in terms of $N$ transmission eigenvalues $T_{i}$ 's of the $N \times N$ matrix $T=t t^{\dagger}$. For example, the dimensionless conductance $G=\operatorname{Tr}\left(t t^{\dagger}\right)=\sum_{i=1}^{N} T_{i}$ [2] and the shot noise $P=\operatorname{Tr}\left[t t^{\dagger}\left(1-t t^{\dagger}\right)\right]=\sum_{i=1}^{N} T_{i}\left(1-T_{i}\right)[3]$. The eigenvalue $0 \leq T_{i} \leq 1$ has a simple physical interpretation as the probability that an electron gets transmitted through the $i$-th channel.

Over the past two decades, the random matrix theory (RMT) has been successfully used [1, 4] to model the transport through such a cavity. Within this RMT approach, one assigns a uniform probability density to all $S$ matrices belonging to the unitary group: this amounts to drawing $S$ at random from one of Dyson's Circular Ensembles [5]. This, in turn, induces a probability measure over the transmission eigenvalues $T_{i}$ 's whose joint probability density (jpd) is known $[1,4,6]$

$$
P\left(\left\{T_{i}\right\}\right)=A_{N} \prod_{j<k}\left|T_{j}-T_{k}\right|^{\beta} \prod_{i=1}^{N} T_{i}^{\beta / 2-1}
$$

where $A_{N}^{-1}=\int_{0}^{1} \ldots \int_{0}^{1} P\left(\left\{T_{i}\right\}\right) \prod_{i} d T_{i}$ is a normalization constant and the Dyson index $\beta$ characterizes different symmetry classes $(\beta=1,2$ according to the presence or absence of time-reversal symmetry and $\beta=4$ in case of spin-flip symmetry). Given this jpd in (1) one is then naturally interested in computing the statistics of transport observables such as the conductance $G$ and the shot noise $P$.

The average and variance of $G$ and $P$ are known $[1,3]$ and in particular for large $N \gg 1,\langle G\rangle=N / 2,\langle P\rangle=$ $N / 8, \operatorname{var}(G)=1 / 8 \beta, \operatorname{var}(P)=1 / 64 \beta$. The fact that the variance of $G$ becomes independent of $N$ for large $N$ has been dubbed the 'universal conductance fluctuations' [1]. In contrast, much less is known for their full distributions. For the conductance, the full distribution is known explicitly only for $N=1$ and $N=2$ [4] and very recently, a solution in terms of Painlevé trascendents for the special case $\beta=2$ has been announced [7]. Also, the behavior of these distributions very close to the endpoints (e.g., near $G=0$ and $G=N)$ have been computed recently [8].

The purpose of this Letter is to present exact results for the full distributions $\mathcal{P}(G, N)$ and $\mathcal{P}(P, N)$ for all $\beta$ in the large $N$ limit. For the conductance $G$, we show that for large $N, \mathcal{P}(G, N) \approx \exp \left(-\frac{\beta}{2} N^{2} \Psi_{G}\left(\frac{G}{N}\right)\right)$ where $\approx$ stands for the precise asymptotic law:

$$
\lim _{N \rightarrow \infty}\left[-\frac{2 \ln \mathcal{P}(N x, N)}{\beta N^{2}}\right]=\Psi_{G}(x)
$$

and the rate function $\Psi_{G}(x)$ is explicitly given in (10). Thus the distribution $\mathcal{P}(G, N)$ has a pure Gaussian form around the mean $\langle G\rangle=N / 2$ over the region $N / 4 \leq G \leq$ $3 N / 4$ and outside this central zone, it has non-Gaussian large deviation tails. The distribution has an extraordinarily weak singularity at the points $G=N / 4$ and $G=3 N / 4$ (only the 3 -rd derivative is discontinuous!). One of our central results is to show that these two weak singularities in the conductance distribution are the direct consequence of two phase transitions in an associated Coulomb gas problem. The distribution of the shot noise $P$ in the allowed range $0 \leq P \leq N / 4$ displays a similar large $N$ behavior: $\mathcal{P}(P, N) \approx \exp \left(-\frac{\beta}{2} N^{2} \Psi_{P}\left(\frac{P}{N}\right)\right)$ 
where $\Psi_{P}(x)$ is given in (12). Once again the central Gaussian regime around the mean $\langle P\rangle=N / 8$ is flanked by two non-Gaussian tails with a weak third order singularities at the transition points $P=N / 16$ and $P=3 N / 16$. In addition to these distributions, we have also computed exactly the large $N$ statistics of the integer moments $\mathcal{T}_{n}=\sum_{i=1}^{N} T_{i}^{n}$. Even though our analytical results are valid for large $N$, we find, rather remarkably, that the numerical results for relatively small values of $N$ (such as $N=4$ ) agree fairly well with our asymptotic results.

We start by demonstrating how the computation of $\mathcal{P}(G, N)$ can be mapped to the calculation of the partition function of a Coulomb gas problem. By definition, $\mathcal{P}(G, N)=\left\langle\delta\left(G-\sum_{i} T_{i}\right)\right\rangle$ where \langle\rangle denotes an average over the jpd in (1). It is then natural to consider the Laplace transform $\left\langle e^{-\beta p N G / 2}\right\rangle=$ $\int \mathcal{P}(G, N) e^{-\beta p N G / 2} d G$, where the Laplace variable $p$ is scaled by the factor $\beta N / 2$ for later convenience. In terms of the eigenvalues $T_{i}$, this Laplace transform can then be expressed as a ratio of two partition functions

$$
\left\langle e^{-\frac{\beta p N}{2} G}\right\rangle=\frac{\int e^{-\frac{\beta p N}{2} \sum_{i} T_{i}} P\left(\left\{T_{i}\right\}\right) \prod_{i} d T_{i}}{\int P\left(\left\{T_{i}\right\}\right) \prod_{i} d T_{i}}=\frac{Z_{p}(N)}{Z_{0}(N)} .
$$

Using (1)

$$
Z_{p}(N)=\int_{0}^{1} \ldots \int_{0}^{1} \exp \left[-\beta E\left(\left\{T_{i}\right\}\right)\right] \prod_{i} d T_{i}
$$

where $E\left(\left\{T_{i}\right\}\right)=\sum_{i}\left(\frac{p N}{2} T_{i}-\frac{(\beta-2)}{2 \beta} \ln \left(T_{i}\right)\right)-$ $\sum_{j<k} \ln \left|T_{j}-T_{k}\right|$. Thus $T_{i}$ 's can be interpreted as the positions of charged particles confined in a 1-d box $[0,1]$, repelling each other via the 2 - $\mathrm{d}$ Coulomb potential (logarithmic) and each subject to an external potential with a linear (with amplitude $p N / 2$ ) and a logarithmic part, the latter being subdominant in the large $N \gg 1$ limit. Then $E$ is the energy of a configuration and $Z_{N}(p)$ is the partition function at an inverse temperature $\beta$.

Our next step is to evaluate the partition function $Z_{p}(N)$ in the large $N$ limit. For that one carries out the multiple integral in (4) in two steps. The first step is a coarse-graining procedure where one sums over (partial tracing) all microscopic configurations of $T_{i}$ 's compatible with a fixed charge density function $\varrho_{p}(T)=$ $N^{-1} \sum_{i} \delta\left(T-T_{i}\right)$ and the second step consists in performing a functional integral over all possible positive charge densities $\varrho_{p}(T)$ that are normalized to unity. Finally the functional integral is carried out in the large $N$ limit by the saddle point method. This procedure has recently been used in the context of the largest eigenvalue distribution of Gaussian [9] and Wishart random matrices [10] and also in other related problems of counting of stationary points in random Gaussian landscapes [11]. Following the general procedure in [9], the resulting func- tional integral, to leading order in large $N$, becomes

$$
Z_{p}(N) \propto \int \mathcal{D}\left[\varrho_{p}\right] e^{-\frac{\beta}{2} N^{2} S\left[\varrho_{p}\right]}
$$

where the action is given by

$$
\begin{aligned}
S\left[\varrho_{p}\right] & =p \int_{0}^{1} \varrho_{p}(T) T d T+B\left[\int_{0}^{1} \varrho_{p}(T) d T-1\right] \\
& -\int_{0}^{1} \int_{0}^{1} d T d T^{\prime} \varrho_{p}(T) \varrho_{p}\left(T^{\prime}\right) \ln \left|T-T^{\prime}\right| .
\end{aligned}
$$

Here $B$ is a Lagrange multiplier enforcing the normalization of $\varrho_{p}(T)$. In the large $N$ limit, one then evaluates the functional integral in (5) by the saddle point method, i.e., one finds the solution $\varrho_{p}^{\star}(T)$ (the equilibrium charge density that minimizes the action or the free energy) from the stationarity condition $\delta S\left[\varrho_{p}\right] / \delta \varrho_{p}=0$ which leads to an integral equation $p T+B=2 \int_{0}^{1} \varrho_{p}^{\star}\left(T^{\prime}\right) \ln \left|T-T^{\prime}\right| d T^{\prime}$. Differentiating once with respect to $T$ leads to a singular integral equation

$$
\frac{p}{2}=\operatorname{Pr} \int_{0}^{1} \frac{\varrho_{p}^{\star}\left(T^{\prime}\right)}{T-T^{\prime}} d T^{\prime}
$$

where Pr denotes the principal part. Assuming one can solve (7) for $\varrho_{p}^{\star}$, one next evaluates the action $S\left[\varrho_{p}\right]$ in (6) at the stationary solution $\varrho_{p}^{\star}$ and then takes the ratio in (3) to get

$$
\left\langle e^{-\beta p N G / 2}\right\rangle \approx e^{-\frac{\beta}{2} N^{2}[\overbrace{\left.S\left[\varrho_{p}^{\star}\right]-S\left[\varrho_{0}^{\star}\right]\right]}^{J_{G}(p)} .}
$$

Inverting the Laplace transform gives the main asymptotic result $\mathcal{P}(G, N) \approx \exp \left(-\frac{\beta}{2} N^{2} \Psi_{G}\left(\frac{G}{N}\right)\right)$ where the rate function is a Legendre transform, $\Psi_{G}(x)=$ $\max _{p}\left[-x p+J_{G}(p)\right]$ with $J_{G}(p)$ given by the free energy difference as in (8).

It then rests to solve (7) to find the equilibrium charge density $\varrho_{p}^{\star}(T)$. Fortunately, singular integral equations such as (7) can be solved in closed form using Tricomi's theorem [12]. Skipping details [13], we find that the solution $\varrho_{p}^{\star}(T)$ has three different forms (see Fig. 1) depending on $p$, the parameter that controls the linear external potential. We get

$$
\varrho_{p}^{\star}(T)=\left\{\begin{array}{l}
\frac{p \sqrt{\frac{4}{p}-T}}{2 \pi \sqrt{T}} ; 0 \leq T \leq \frac{4}{p} ; \quad p \geq 4 \\
\frac{p\left[\frac{4+p}{2 p}-T\right]}{2 \pi \sqrt{T(1-T)}} ; 0 \leq T \leq 1 ; \quad-4 \leq p \leq 4 \\
\frac{|p| \sqrt{T-\left(1-\frac{4}{|p|}\right)}}{2 \pi \sqrt{1-T}} ; 1-\frac{4}{|p|} \leq T \leq 1 ; \quad p \leq-4
\end{array}\right.
$$


Thus the equilibrium charge density undergoes two phase transitions as one tunes $p$ respectively at $p=4$ and $p=$ -4 . For $p \geq 4$, the linear potential is strong enough to confine the charges near $T=0$ leading to an inverse square root divergence of the density at $T=0$ and the density becomes zero beyond $4 / p$. As $p$ approaches the critical value 4 from above, the upper edge of the density support approaches the maximum value 1 and for $-4 \leq$ $p \leq 4$ the charges are spread over the full box $[0,1]$ with the density diverging at the two end points. Finally for $p \leq-4$, charges accumulate on the right wall $T=1$ of the box with the density vanishing for $T \leq 1-4 /|p|$. Note that the equilibrium density (in the large $N$ limit) is independent of $\beta$. The analytical solutions in the 3 regimes above are depicted in Fig. 1 together with MC results [14].
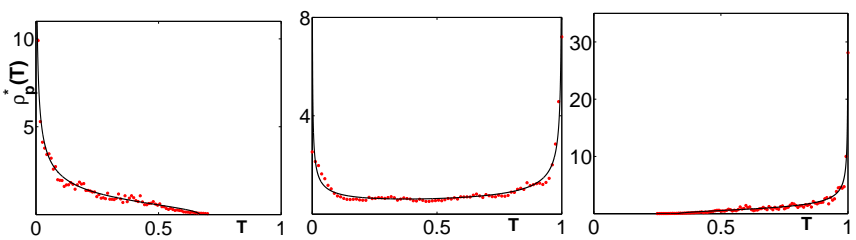

FIG. 1: Charge density $\varrho_{p}^{\star}(T)$ for conductance (numerical, in red dots; theory, in solid black line). From left to right $p=6,-1,-6$. The numerical density [14] is for $N=5$ and $\beta=2$.

Inserting $\varrho_{p}^{\star}(T)$ in the action (6) one obtains the free energy difference in (8)

$$
J_{G}(p)= \begin{cases}3 / 2+p+\ln (-p / 4) & p \leq-4 \\ -\frac{p^{2}}{32}+\frac{p}{2} & -4 \leq p \leq 4 \\ 3 / 2+\ln (p / 4) & p \geq 4\end{cases}
$$

This analytical prediction is compared in Fig. 2 with MC simulations performed using similar tricks as in the case of $\varrho_{p}^{\star}(T)$. Finally, the rate function $\Psi_{G}(x)=\max _{p}[-x p+$ $\left.J_{G}(p)\right]$ in (2) is then given by (see Fig. 2):

$$
\Psi_{G}(x)= \begin{cases}\frac{1}{2}-\ln (4 x) & 0 \leq x \leq \frac{1}{4} \\ 8\left(x-\frac{1}{2}\right)^{2} & \frac{1}{4} \leq x \leq \frac{3}{4} \\ \frac{1}{2}-\ln [4(1-x)] & \frac{3}{4} \leq x \leq 1\end{cases}
$$

Using the quadratic form in $1 / 4 \leq x=G / N \leq 3 / 4$ above in $\mathcal{P}(G, N) \approx \exp \left(-\frac{\beta}{2} N^{2} \Psi_{G}\left(\frac{G}{N}\right)\right)=e^{-4 \beta\left(G-\frac{N}{2}\right)^{2}}$ gives the Gaussian form in this central region from which one easily reads off the well known values for mean and variance $\langle G\rangle=N / 2$ and $\operatorname{var}(G)=1 / 8 \beta$. On the other hand, near the two endpoints $x=0$ and $x=1$, using $\Psi_{G}(x) \sim-\ln (x)$ and $\sim-\ln (1-x)$ as in (10), we get the power law dependence, $\mathcal{P}(G, N) \sim G^{\beta N^{2} / 2}$ (as $G \rightarrow 0)$ and $\mathcal{P}(G, N) \sim(N-G)^{\beta N^{2} / 2}($ as $G \rightarrow N)$ which are in agreement, to leading order in large $N$, with the exact far tails obtained in [8]. The central Gaussian regime matches smoothly with the two side regimes (only the third derivative is discontinuous). Such weak nonanaliticities of third order were also recently found in the entropy distribution in a quantum entanglement problem [15]. An expression similar to (10) was recently found in [7] by a different method. However, the intermediate regime with an exponential decay claimed in [7] does not appear in our solution.

The same formalism can be easily extended to find the distribution $\mathcal{P}(P, N)$ of the shot noise $P=\sum_{i=1}^{N} T_{i}(1-$ $\left.T_{i}\right)$. It turns out to be convenient to work with the shifted variable $Q=N / 4-P=\sum_{i=1}^{N}\left(1 / 2-T_{i}\right)^{2}$. Considering the Laplace transform of the distribution of $Q$, we then get a new Coulomb gas where the charges, i.e., the shifted eigenvalues $\mu_{i}=1 / 2-T_{i}$ 's are confined in the box $[-1 / 2,1 / 2]$, repel each other logarithmically and each experiences an external harmonic (with amplitude proportional to the Laplace variable $p$ ) +logarithmic potential. The equilibrium charge density $\varrho_{p}^{\star}(\mu)$ of this Coulomb gas can be computed again by solving the integral equation $p \mu=\operatorname{Pr} \int_{-1 / 2}^{1 / 2} \frac{\varrho_{p}^{*}\left(\mu^{\prime}\right)}{\mu-\mu^{\prime}} d \mu^{\prime}$. Skipping details [13], we again find two phase transitions upon tuning the value of $p$. For $p>8$, the density has a semi-circular form over $\mu \in[-\sqrt{2 / p}, \sqrt{2 / p}]$. For $-8<p<8$, the density has square root divergences at the box ends $\mu= \pm 1 / 2$. For $p<-8$, the density breaks up into two disjoint sectors around the two endpoints and vanishes in the middle of the box (see Fig. 3). In these three regions $p>8$, $-8<p<8$ and $p<-8$ the exact form of the density is given by

$$
\varrho_{p}^{\star}(\mu)=\left\{\begin{array}{l}
\frac{p}{\pi} \sqrt{\frac{2}{p}-\mu^{2}} ; \quad-\sqrt{\frac{2}{p}} \leq \mu \leq \sqrt{\frac{2}{p}} \\
\frac{p}{\pi \sqrt{1 / 4-\mu^{2}}}\left[\frac{8+p}{8 p}-\mu^{2}\right] ; \quad-\frac{1}{2} \leq \mu \leq \frac{1}{2} \\
\frac{|p \mu| \sqrt{\mu^{2}-\zeta_{p}^{2}}}{\pi \sqrt{1 / 4-\mu^{2}}} ; \quad\left|\zeta_{p}\right| \leq|\mu| \leq \frac{1}{2}
\end{array}\right.
$$

where $\zeta_{p}=\sqrt{1 / 4-2 /|p|}$ and clearly $\left|\zeta_{p}\right|<1 / 2$ for $p<$ -8 . In the last case, $\varrho_{p}^{\star}(\mu)=0$ for $-\zeta_{p}<\mu<\zeta_{p}$.

Following steps similar to the conductance case, we then compute the free energy difference associated with the shifted shot noise $Q=N / 4-P$

$$
J_{Q}(p)= \begin{cases}\frac{3}{4}+\frac{1}{2} \ln \left(\frac{p}{8}\right) & p \geq 8 \\ \frac{-p^{2}}{256}+\frac{p}{8} & -8 \leq p \leq 8 \\ \frac{3-|p|}{4}+\frac{1}{2} \ln \left(\frac{|p|}{8}\right) & p \leq-8\end{cases}
$$

from which the rate function for the unshifted shot noise $P=\sum_{i} T_{i}\left(1-T_{i}\right)$ can be computed. Noting that $0 \leq$ 
$T_{i} \leq 1$ implies that the scaled shot noise $0 \leq x=P / N \leq$ 1/4, we get (see Fig. 2):

$$
\Psi_{P}(x)= \begin{cases}\frac{1}{4}-\frac{1}{2} \ln (16 x) & 0 \leq x \leq \frac{1}{16} \\ 64\left(\frac{1}{8}-x\right)^{2} & \frac{1}{16} \leq x \leq \frac{3}{16} \\ \frac{1}{4}-\frac{1}{2} \ln \left[16\left(\frac{1}{4}-x\right)\right] & \frac{3}{16} \leq x \leq \frac{1}{4}\end{cases}
$$

Once again, the rate function $\Psi_{P}(x=P / N)$ is weakly nonanalytic at the critical values $x=P / N=1 / 16,3 / 16$ where a central Gaussian regime connects the two nonGaussian regimes on either sides. From the central regime, one again reads off the mean and the variance of the shot noise for large $N:\langle P\rangle=N / 8$ and $\operatorname{var}(P)=1 / 64 \beta$. At the two endpoints $P \rightarrow 0$ and $P \rightarrow N / 4$, we get using (12), power law behavior for the distribution, $\mathcal{P}(P, N) \sim P^{\beta N^{2} / 4}$ (as $P \rightarrow 0$ ) and $\mathcal{P}(P, N) \sim(N / 4-P)^{\beta N^{2} / 4}$ (as $\left.P \rightarrow N / 4\right)$. The latter limit is in agreement with the far tail result of [8], while the precise asymptotics near $P \rightarrow 0$ was not known before.
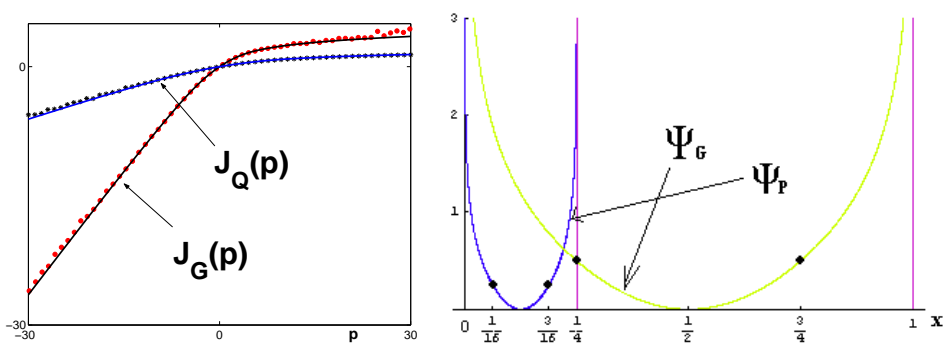

FIG. 2: Left: Free energy difference $J_{G}(p)$ (red) and $J_{Q}(p)$ (blue) from Montecarlo (MC) simulations and theory (solid line). We have used $-30 \leq p \leq 30, \beta=2$ and $N=5$ (for conductance) and $N=4$ (for shot noise) respectively. Right: Rate functions $\Psi_{G}(x)$ (green) and $\Psi_{P}(x)$ (blue) (see (10) and (12)). The black dots indicate the two critical points on each curve.

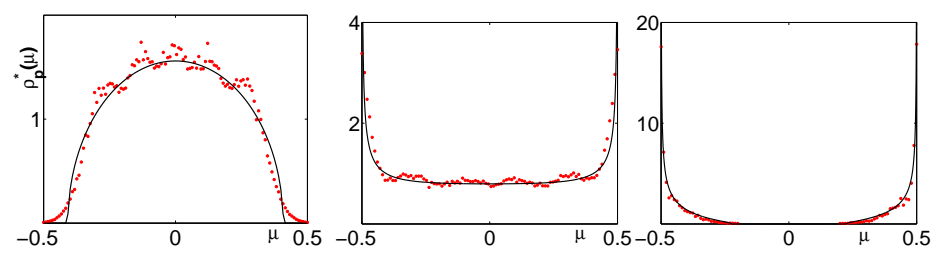

FIG. 3: Charge density $\varrho_{p}^{\star}(\mu)$ for the shifted shot noise $Q$. From left to right $p=12,2,-10$.

The large- $N$ behavior (2) is generic for the distribution of any linear statistics on the transmission eigenvalues (of the form $\sum_{i} a\left(T_{i}\right)$ where $a(T)$ can be nonlinear as in the case of shot noise) and the large- $N$ formalism developed here may, in principle, be extended to compute the distribution of any arbitrary linear statistic $a(T)$. In particular, as a byproduct one can derive the mean and variance of any linear statistics by computing the central quadratic behavior of the associated rate function $\Psi(x)$. In certain cases this provides new explicit formulae for mean and variance that are not easily accessible by other general methods such as in [16]. As an example we consider briefly the case of integer moments $\mathcal{T}_{n}=\sum_{i=1}^{N} T_{i}^{n}$, relevant for statistics of charge cumulants $[8,17,18]$. The rate function close to the maximum (Gaussian regime) is given by [13]: $\Psi_{\mathcal{T}_{n}}(x)=\left(4 A_{n}\right)^{-1}\left(x-B_{n}\right)^{2} \quad$ for $x_{n}^{(-)}<x<x_{n}^{(+)}$, where $x_{n}^{( \pm)}$are $n$-dependent edge points and the constants $A_{n}$ and $B_{n}$ are given by $A_{n}=(2 n-1) \Gamma(n+$ $1 / 2) \Gamma(n-1 / 2) / 16 \pi n \Gamma^{2}(n)$ and $B_{n}=4^{-n}\left(\begin{array}{c}2 n \\ n\end{array}\right)$.

This implies: $\mathcal{P}\left(\mathcal{T}_{n}, N\right) \approx \exp \left[-\frac{\beta}{8 A_{n}}\left(\mathcal{T}_{n}-B_{n} N\right)^{2}\right]$ near the mean. From this Gaussian form one can read off the mean and the variance. For the mean we find a $\beta$-independent result $\left\langle\mathcal{T}_{n}\right\rangle=B_{n} N=\frac{N}{4^{n}}\left(\begin{array}{c}2 n \\ n\end{array}\right)$. In the special case $\beta=2$ this was recently proved in [18] by other methods. For the variance we find a new exact result: $\operatorname{var}\left(\mathcal{T}_{n}\right)=\frac{4 A_{n}}{\beta}=\frac{1}{4 \beta \pi} \frac{(2 n-1) \Gamma(n+1 / 2) \Gamma(n-1 / 2)}{n \Gamma^{2}(n)}$ which reduces to the conductance result $1 / 8 \beta$ for $n=1$ and approaches to the universal constant $1 / 2 \beta \pi$ as $n \rightarrow \infty$. Our new result for $\operatorname{var}\left(\mathcal{T}_{n}\right)$ for $N \gg 1$ and its universal asymptote cannot be obtained easily from the formula in [18], valid for a finite number of open channels.

In summary, we have obtained exact analytical distributions for the conductance and the shot noise for a mesoscopic cavity with two leads and $N$ channels each in the large $N$ limit. Our results reveal a rich thermodynamic behavior including two phase transitions in an associated Coulomb gas problem leading to extraordinarily weak nonanaliticities in the distributions. We believe that the Coulomb gas method (well suited for large $N$ ) used here is very general and will be useful in other problems as well.

Acknowledgments: PV acknowledges support from Marie Curie Early Stage Fellowship (NET-ACE project). Helpful discussions with M. Novaes, D. Savin, G. Akemann and L. Shifrin are also gratefully acknowledged.

[1] C.W.J. Beenakker, Rev. Mod. Phys. 69, 731 (1997).

[2] R. Landauer, IBM J. Res. Dev. 1, 223 (1957) and Phil. Mag. 21, 863 (1970); D.S. Fisher and P. A. Lee, Phys. Rev. B 23, 6851 (1981).

[3] Ya.M. Blanter and M. Büttiker, Phys. Rep. 336, 1 (2000).

[4] H.U. Baranger and P.A. Mello, Phys. Rev. Lett. 73, 142 (1994); R.A. Jalabert, J.-L. Pichard and C.W.J. Beenakker, Europhys. Lett. 27, 255 (1994); M.H. Pedersen, S.A. van Langen and M. Büttiker, Phys. Rev. B 57, 1838 (1998); A. García-Martín and J.J. Sáenz, Phys. Rev. Lett. 87, 116603 (2001).

[5] M.L. Mehta, Random Matrices, 3rd Edition, (ElsevierAcademic Press) (2004).

[6] P.J. Forrester, J. Phys. A: Math. Gen. 39, 6861 (2006). 
[7] V.Al. Osipov and E. Kanzieper, [arXiv:0806.2784] (2008).

[8] H.-J. Sommers, W. Wieczorek and D.V. Savin, Acta Phys. Pol. A 112, 691 (2007).

[9] D.S. Dean and S.N. Majumdar, Phys. Rev. Lett. 97, 160201 (2006); Phys. Rev. E 77, 041108 (2008).

[10] P. Vivo, S.N. Majumdar and O. Bohigas, J. Phys. A: Math. Theor. 40, 4317 (2007).

[11] A.J. Bray and D.S. Dean, Phys. Rev. Lett. 98, 150201 (2007); Y.V. Fyodorov, H-J. Sommers and I. Williams, JETP Letters 85, 261 (2007); Y.V. Fyodorov and I. Williams, J. Stat. Phys. 129, 1081 (2007).

[12] F.G. Tricomi, Integral Equations, (Pure Appl. Math V, Interscience, London, 1957).

[13] details will be published elsewhere.

[14] Noting that the average density is the same as the single point marginal of a joint density of $N$ variables, it can be expressed as the ratio (for simplicity we choose $\beta=2) \varrho_{p}^{\star}\left(x_{1}\right)=\left\langle e^{-p N \sum_{i=1}^{N} x_{i}} \prod_{j<k}\right| x_{j}-$
$\left.\left.x_{k}\right|^{2}\right\rangle_{N-1} /\left\langle e^{-p N \sum_{i=1}^{N} x_{i}} \prod_{j<k}\left|x_{j}-x_{k}\right|^{2}\right\rangle_{N}$ where the average is taken over $N-1$ random numbers $x_{2}, \ldots, x_{N}$ (numerator) and $N$ (denominator) with a flat measure over $[0,1]$, with $x_{1}$ spanning the interval $(0,1)$. The numerator and the denominator are then separately evaluated numerically.

[15] P. Facchi, U. Marzolino, G. Parisi, S. Pascazio and A. Scardicchio, Phys. Rev. Lett. 101, 050502 (2008).

[16] C.W.J. Beenakker, Phys. Rev. Lett. 70, 1155 (1993).

[17] P.W. Brouwer and C.W.J. Beenakker, J. Math. Phys. 37, 4904 (1996); D.V. Savin and H.-J. Sommers, Phys. Rev. B 73, 081307(R) (2006); P. Vivo and E. Vivo, J. Phys. A: Math. Theor. 41, 122004 (2008).

[18] M. Novaes, Phys. Rev. B 75, 073304 (2007); ibid 78, 035337 (2008). 